\title{
ANÁLISE DO CONSUMO ALIMENTAR DE PROFESSORES E A PREVALÊNCIA DE ALTERAÇÕES INTESTINAIS NO CONTEXTO DA PANDEMIA PELO COVID-19
}

\author{
ANALYSIS OF TEACHERS' FOOD CONSUMPTION AND THE PREVALENCE OF \\ INTESTINAL ALTERATIONS IN THE CONTEXT OF THE COVID-I9 PANDEMIC
}

\author{
Bianca de Aguiar Cabral ${ }^{1}$ \\ Irene Coutinho de Macedo ${ }^{2}$ \\ Lis Proença Vieira ${ }^{3}$
}

RESUMO: Introdução: A pandemia pelo covid-ı9 implicou em alterações no estilo de vida da população, especialmente entre os professores, cuja rotina foi impactada por novas metodologias de trabalho. Este cenário pode favorecer a mudanças no padrão de consumo alimentar e alterações no funcionamento intestinal. Objetivo: Identificar alterações no funcionamento intestinal e o consumo alimentar de professores no período de execução de trabalho remoto durante a pandemia. Material e métodos: Estudo transversal com professores convidados a responderem um questionário online a partir de convites via Whats $A p p$ e redes sociais, que abordaram questões sobre os marcadores de alimentação saudável e funcionamento intestinal, tais como frequência e consistência das fezes. Resultados: Participaram do estudo 43 professores, média de idade de 46 anos, 88,4\% do sexo feminino, 72,1\% atuando no ensino fundamental e todos da rede pública. Foi observado baixo consumo de água 86,04\%; de feijão 81,39\%; de alimentos industrializados $76,74 \%$ e de hortaliças $51,16 \%$. A maioria da apresentou um consumo adequado de frutas $(53,48 \%)$ e elevado de doces (72,09\%). Quanto às alterações intestinais, $7 \%$ mantiveram o quadro prévio de constipação intestinal, 4,65\% apresentaram risco de desenvolvê-lo e $7 \%$ apresentaram alterações na frequência e/ou consistência das fezes. Apesar do número reduzido de casos, a presença de constipação foi associada com o baixo consumo de feijão e elevado consumo de alimentos industrializados ( $p<0,05$ ). Conclusão: Os professores estudados apresentaram baixa prevalência de constipação e de alterações intestinais e a maioria apresentou consumo adequado de frutas e baixo consumo de industrializados, mas ingestão inadequada, principalmente, de água, feijão e doces.

Palavras-chave: I. Docentes 2. Constipação Intestinal 3. Consumo alimentar.

\footnotetext{
${ }^{\mathrm{I}}$ Bacharelado em Nutrição com bolsa de iniciação científica pelo Centro Universitário Senac Santo Amaro. São Paulo, SP, Brasil. E-mail: biancadeaguiarcabral@yahoo.com.br

${ }^{2}$ Coordenadora do Bacharelado em Nutrição do Centro Universitário Senac Santo Amaro. São Paulo, SP, Brasil. Mestre em Nutrição Humana Aplicada pela Universidade de São Paulo. E-mail: irene.cmacedo@sp.senac.br.

${ }^{3}$ Docente do Bacharelado em Nutrição do Centro Universitário Senac Santo Amaro. São Paulo, SP, Brasil. Mestre e Doutora em Ciências pela Universidade de São Paulo. E-mail: lis.pvieira@sp.senac.br
} 
ABASTRACT: Introduction: The covid-19 pandemic resulted in changes in the population's lifestyle, especially among teachers, whose routine was impacted by new work methodologies. This scenario can favor changes in the pattern of food consumption and changes in bowel function. Objective: To identify changes in bowel function and food consumption of teachers during the period of remote work during the pandemic. Material and methods: Cross-sectional study with professors invited to answer an online questionnaire from invitations via WhatsApp ${ }^{\circledR}$ and social networks that addressed questions about the markers of healthy eating and bowel function, such as frequency and consistency of stools. Forty-three teachers participated in the study, mean age 46 years, 88.4\% female, $72.1 \%$ working in elementary school and all from the public network. Results: It was observed low water consumption 86.04\%; of beans 81.39\%; of processed foods $76.74 \%$; of vegetables $51.16 \%$. There was a regular consumption of fruit $53.48 \%$ and a high consumption of sweets $72.09 \%$. As for intestinal changes, $7 \%$ maintained the previous picture of intestinal constipation and $4.65 \%$ were at risk of developing it and $7 \%$ showed changes in the frequency and/or consistency of stools. The presence of constipation was associated with low consumption of beans and high consumption of processed foods ( $p<0.05$ ), despite the small number of cases. Conclusion: The teachers studied had a low prevalence of constipation and intestinal disorders and most had adequate consumption of fruits and low consumption of industrialized products, but inadequate consumption, especially regarding the consumption of water, beans and sweets

Keywords: I. Faculty 2. Constipation 3. Food Consumption.

\section{INTRODUÇÃO}

A pandemia pelo coronavírus (Covid-19) no ano de 2020 acarretou mudanças de comportamento e atitudes em nível global a fim de reduzir o contágio pelo vírus (DEMOLINER;DALTÓE, 2020). No entanto, as repercussões a partir de tais mudanças ocorreram de formas diferentes conforme cada configuração intercultural (PONTI et al.,2020).

Segundo Malta et al (2020) e Ponti et al (2020), tratando-se do cenário brasileiro, o Ministério da Saúde propôs medidas higiênicas de alcance individual tais como higienização das mãos e uso de máscaras, assim como medidas de alcance comunitário, como evitar formas de aglomerações, proibição do funcionamento de escolas e universidades locais de convívio, no qual destaca-se a restrição social que foi a medida mais difundida pelas autoridades a fim evitar a disseminação do vírus em larga escala.

Essas recomendações implicaram em mudanças no estilo de vida da população, muitas vezes em contradição ao discurso de representantes governamentais, que também corroborou para o agravo na saúde mental da população, desencadeando elevações no nível de estresse acompanhado de desordens físicas como perturbações do sono, dores no estômago, entre outras (PONTI et al.,2020).

Ainda segundo Malta et al (2020) como consequência do isolamento social houve também uma redução no nível de atividade física observada em nível nacional. A partir de 
outro estudo realizado por Maynard et al (2020), observou-se uma associação do baixo nível de atividade física com um maior grau de ansiedade, a qual impactou no padrão de consumo alimentar na população adulta apresentando um aumento na ingestão de alimentos ricos em açúcar. Essa evidencia fortalece o exposto pela literatura de que o consumo alimentar é influenciado por aspectos de ordem psíquica, como a ansiedade e a depressão, os quais instigam o indivíduo a buscar alimentos mais palatáveis como aqueles ricos em açúcar e gorduras (ALVERENGA, 2019; NOLAN; JENKINE, 2019; SARAIVA, 2019).

A inadequação alimentar pode aumentar o risco de desenvolver doenças crônicas não transmissíveis (DCNTS) por diferentes mecanismos (WHO, 2003), que podem estar associados a modificações na saúde de órgãos vitais como o intestino, por seu envolvimento nos processos de digestão, absorção de nutrientes, síntese de substâncias, a exemplo de enzimas, vitaminas $k$ e biotina e participação no sistema imune, auxiliando no combate a doenças infecciosas (OLIVEIRA et al., 2020).

Entende-se que uma boa saúde intestinal também está fortemente relacionada ao equilíbrio entre bactérias benéficas e patógenas, sendo as bactérias benéficas grandes responsáveis por auxiliar na manutenção da integridade e funcionalidade do órgão (PASSOS; MORAES FILHO, 2017).

Todavia os fatores já mencionados: consumo alimentar, nível de atividade física e saúde mental interferem fortemente na regulação do funcionamento intestinal (OLIVEIRA et al., 2020). Em relação ao cenário trabalhista, algumas categorias profissionais, dentre elas a docência tanto em rede pública quanto privada, sofreram maior impacto frente as medidas de isolamento social. Foram necessárias adaptações das atividades escolares para que ocorressem de forma remota home-office. Este foi um grande desafio para os profissionais tendo em vista que grande parte deles não estavam familiarizados com as ferramentas digitais, além da insegurança quanto a eficácia dessa metodologia de ensino a distância e falta de planejamento de ações de médio a longo prazo pelos gestores das instituições de ensino (CRUZ et al.,2020).

$\mathrm{Na}$ literatura há dados de prejuízos na saúde física e mental dos professores após a mudança na rotina de trabalho (CRUZ et al.,2020), mas não traz informações sobre sua saúde intestinal associada ao consumo alimentar. Considerando que esta população foi uma das mais afetadas pelas incertezas e alterações na metodologia de trabalho, que dá margem para mudanças no padrão de consumo alimentar e repercussão na saúde intestinal, este trabalho teve como objetivo identificar alterações no funcionamento intestinal e o consumo alimentar de professores durante a pandemia.

\section{MATERIAL E MÉTODOS}

Foi realizada uma pesquisa de campo do tipo transversal compreendendo dados de natureza qualitativa e quantitativa.

Foram convidados a participar da pesquisa professores da rede pública ou privada de todas as modalidades de ensino, com idade de 20 a 59 anos, da cidade de São Paulo. Foi considerada uma amostra de conveniência, ou seja, o número de indivíduos dependeu da disponibilidade e adesão daqueles que aceitaram participar da pesquisa (FREITAS, 200o). Foram excluídos professores que estivessem exercendo sua atividade exclusivamente de 
modo presencial ou que já apresentassem diagnóstico de doenças intestinais. Todos preencheram um Termo de Consentimento Livre e Esclarecido, de acordo com as normas de pesquisa científica da Resolução N.ㅇ 510, de o7 de abril de 2016 do Conselho Nacional de Saúde. O projeto de pesquisa foi aprovado pelo Comitê de ética sob o número CAAE 48789821.7 .0000 .8054 .

A divulgação da pesquisa foi por meio do aplicativo Whats app ${ }^{\circledR}$ e redes sociais das pesquisadoras. Os dados foram coletados por meio de um questionário online da plataforma Google forms ${ }^{\circledR}$, durante o mês de agosto de 2021, após aprovação pelo comitê de ética em pesquisa. $O$ questionário continha questões abrangendo a regularidade do funcionamento intestinal e do padrão de consumo alimentar a partir dos marcadores saudáveis: frutas, verduras e legumes e feijão e não saudáveis: doces e alimentos industrializados.

As questões relacionadas ao consumo alimentar foram baseadas no questionário Convid do Instituto de Pesquisa Fiocruz Malta et al (2020), segundo o qual considerou-se um consumo adequado de frutas; verduras; legumes e feijão caso aparecessem em cinco ou mais dias na semana. O consumo de alimentos comprados congelados prontos para consumo (industrializados) e doces foi considerado inadequado caso tivesse aparecido duas ou mais vezes na semana. Para os dados sobre a ingestão de água foi considerada adequada a ingestão de 2 Litros para as mulheres e 2,5 Litros para os homens (EFSA, 2010).

As alterações no funcionamento intestinal foram caracterizadas quanto a frequência de evacuação, consistência das fezes e presença de constipação. As perguntas foram adaptadas a partir do modelo existente para verificação da saúde intestinal pelo critério Roma III da World Gastroenterology Organization (WGO, 2010). O questionário teve como objetivo rastrear a constipação intestinal e seguiu os seguintes critérios: menos de três vezes de evacuação na semana (geral) e ao menos dois dos específicos: dor ao evacuar, uso de manobras como exemplo o uso de laxantes, fezes endurecidas ou fragmentadas, sensação de fezes incompletas, durante um período de três meses consecutivos. Para identificar as alterações na consistência das fezes e frequência semanal de evacuação, as questões se remeteram aos períodos antes de home office e o atual.

Os dados quantitativos foram apresentados na forma de frequência absoluta e relativa e a análise de associação das variáveis qualitativas foi realizada pelo teste Qui quadrado, no qual foi considerado significante se $\mathrm{p}<0,05$. Os testes estatísticos foram realizados a partir do software JAMOVI ${ }^{\circledR}$.

\section{RESULTADOS E DISCUSSÃO}

Obteve-se 52 questionários respondidos, dos quais foram excluídos 9, sendo 4 por apresentarem doenças gastrointestinais prévias, I pela idade do participante não atender aos critérios de idade e 4 por não terem realizado home office, totalizando 43 questionários.

A amostra foi representada por $38(88,4 \%)$ do sexo feminino. A modalidade de ensino foi representada por $\mathrm{I} 6(37,20 \%)$ participantes do ensino fundamental II; $15(34,88 \%)$ do ensino fundamental I; 8 (I8,60\%) do ensino médio e 4 (9,30\%) do ensino infantil, ou seja, em sua maior parte trabalhando na educação básica, sendo todos atuantes em escolas públicas. A 
idade mínima na amostra foi 32 anos e a máxima 59 anos, sendo a média de idade de 46,27 anos. No momento da pesquisa $39(90,69 \%)$ dos professores responderam que haviam feito home office, mas já estavam voltando ao trabalho presencial.

A representatividade feminina se justifica por fatos históricos no século XIX cuja presença de mulheres na educação ocorreu inicialmente em escolas improvisadas voltadas a cuidados e orientações para crianças (INEP, 2018; FERREIRA, 1998) e, posteriormente no século $X X$, a sua inserção no magistério com a institucionalização do ensino primário, passando a compor o quadro de servidoras públicas (INEP, 2018; LEAL, 2016).

Com relação ao consumo alimentar, observou-se que a maioria da amostra apresentou uma ingestão adequada de frutas e baixa de alimentos industrializados (tabela I), o que vai de encontro com os princípios de uma alimentação saudável (BRASIL, 2014).

O consumo regular de frutas pelos professores é semelhante aos resultados obtidos por Cheffer e Micaliski (2017) e o baixo consumo de ultraprocessados é reforçado pelo estudo de Gallina et al (2013), que encontrou $72,2 \%$ da sua amostra de 79 professores, também predominantemente do sexo feminino com o consumo de industrializados menos que duas vezes por semana. Da mesma forma Landim et al (2021) demonstrou numa amostra de 43 professores uma prevalência de $62,8 \%$ de baixo consumo de alimentos industrializados.

O mesmo não observou associação entre o conhecimento nutricional dos professores com com o seu consumo alimentar.

No presente estudo observou-se que $37(86,04 \%)$ dos professores apresentaram ingestão inadequada de água, 35 (81,39\%) de feijão, 31 (72,09\%) de doces, 22 (51,16\%) de verduras e legumes (tabela I). Segundo a Associação Brasileira de Nutrição (ASBRAN, 202I), as percepções de professoras de educação básica são limitadas, pois não relacionam como o baixo consumo de industrializados e um maior consumo de frutas, verduras e legumes (FLV's) podem repercutir biologicamente, o que poderia ser um dos fatores limitantes para a busca de um consumo alimentar mais saudável. Com relação ao consumo do feijão e outras leguminosas os resultados obtidos diferem de Landim (2021) o qual encontrou $58,1 \%$ de adequação na sua amostra, entretanto o mesmo não deixou claro o critério para a frequência considerada adequada.

Tabela I. Adequação dos marcadores de consumo alimentar entre os professores estudados $(n=43)$. São Paulo (SP), 2021.

\begin{tabular}{l|cc} 
Marcadores de consumo alimentar & $\boldsymbol{n}$ & $\%$ \\
\hline Fruta (5 ou mais vezes na semana) & 23 & 53,48 \\
Verduras e legumes (5 ou mais vezes na semana) & $2 \mathrm{I}$ & 48,83 \\
Feijão (5 ou mais vezes na semana) & 8 & 18,60 \\
Doce (menos que 2 vezes na semana) & II & 25,58 \\
Industrializados (menos que 2 vezes na semana) & 33 & 76,74 \\
Água (2 L para mulheres e 2,5 L para homens) & 6 & 13,95
\end{tabular}

A análise das alterações intestinais mostrou que 3 (7\%) da amostra apresentou quadro de constipação segundo os critérios da pesquisa e 2(4,65\%) apresentaram sintomas parciais, que podem indicar risco para o desenvolvimento desse quadro, tais como consistência das fezes fragmentadas ou duras com aspecto escurecido, sensação de fezes incompletas e uso de probióticos, dores ao evacuar e/ou uso de laxantes. Além disso 3 
(7\%) informaram apenas alterações na consistência e/ou frequência de evacuação sem utilização de manobras, sendo que um destes indicou uma alteração positiva com melhora nos critérios de avaliação.

Tanto a constipação quanto o risco para a mesma ocorreram apenas no público feminino. A maior prevalência de constipação no sexo feminino se justifica por questões hormonais como a ação da progesterona que se encontra mais elevada na fase lútea do ciclo menstrual ou durante o segundo e o terceiro trimestre de uma gestação, bem como os danos à musculatura do assoalho pélvico durante cirurgias ginecológicas e obstétricas. Além do sexo, a constipação é uma patologia multifatorial, sendo influenciada por aspectos neurológicos, uso de medicamentos, dietas inadequadas com baixo consumo de fibras, idade, baixo nível socioeconômico, baixa escolaridade, disfunções endócrinas e metabólicas, inatividade física, distúrbios psiquiátricos e causas idiopáticas (JAQUES et al., 2020).

O uso de laxantes e outros medicamentos precisa ser avaliado com cautela, pois o uso indiscriminado destes produtos é responsável por alterações motoras e secretivas no trato gastrointestinal, podendo produzir tanto a própria constipação, como diarreia, além de desidratação e má nutrição (AMARAL et al., 2012).

$\mathrm{Um}$ dos fatores que podem estar associados a alterações gastrointestinais são as mudanças na rotina, pois podem favorecer a geração de estresse. O estresse físico e/ ou psicológico são aceitos como disparadores de distúrbios gastrointestinais, no caso específico do intestino pode ocorrer alteração da permeabilidade de barreira favorecendo a reações adversas como diarreia, absorção indesejada de patógenos intestinais entre outras. Um fator importante que deve ser observado a magnitude e cronicidade do estresse a fim de evitar que se perpetuem tais alterações favorecendo ao surgimento de outras patologias (LAUFFER, 2015). No entanto, todos os casos identificados como constipação, mantiveram as características de consistência das fezes duras com aspecto escurecido e menos de três evacuações na semana desde antes da alteração de trabalho para o home office.

Apesar das mudanças ocorridas na pandemia, não foi possível observar uma associação entre os hábitos alimentares atuais e a presença de constipação, exceto naqueles com o consumo inadequado de feijão e de industrializados $(\mathrm{p}<0,05)$, provavelmente devido à baixa prevalência dos quadros de constipação (tabela 2). Todavia nota-se que duas das mulheres consideradas constipadas apresentaram todos os itens de consumo alimentar e ingestão de água inadequados. Vale ressaltar que o consumo alimentar atual não reflete o desenvolvimento de doenças intestinais, assim como de outras doenças crônicas, que dependem de hábitos de vida de longo prazo (DUNCAN et al., 2012).

Tabela 2. Associação da inadequação do consumo alimentar e ingestão hídrica e a presença de constipação. São Paulo (SP), 2021

\begin{tabular}{l|cc} 
Itens de Inadequação & Constipados $n(\%)$ & $n$ \\
\hline Fruta (<5 vezes na semana) & 3 (100) & 0,054 \\
Verduras e legumes $(<5$ vezes na semana) & 3 (100) & 0,079
\end{tabular}


Feijão (< 5 vezes na semana)

Doce (>2 vezes na semana)

Industrializados (>2 vezes na semana)

Água (2Litros para mulheres e 2,5Litros para homens)

\begin{tabular}{|cc}
$2(66,66)$ & 0,049 \\
$2(66,66)$ & 0,077 \\
$3(100)$ & 0,001 \\
$3(100)$ & 0,470
\end{tabular}

Normalmente, a baixa ingestão de água e de fibras predispõem a constipação. As fibras estão mais presentes em alimentos in natura e minimamente processados como o caso do feijão, frutas, verduras e legumes e, juntamente com água, promovem a formação das fezes e estimulam a motilidade intestinal (BRASIL, 20I4; CUPPARI, 20I9; OLIVEIRA, 2019).

Estima-se que só pela respiração há uma perda hídrica diária de $250 \mathrm{~mL}$ a $600 \mathrm{~mL}$, portanto a ingestão de água é essencial para o equilíbrio de fluidos corporais como a saliva, secreções e sangue além de participar de funções orgânicas como a manutenção da temperatura corporal e transporte de nutrientes para as células (SBAN, 2016). Segundo Carvalho e Zanardo (2010), a ingestão de água no público adulto tende a ser baixa, devido a substituição por outros líquidos, principalmente acompanhando as grandes refeições.

Os alimentos processados, diferentemente dos alimentos minimamente processados e in natura, como exemplo as verduras e legumes, além de conter ingredientes adicionais, que alteram o valor nutricional do alimento, nem sempre apresentam uma quantidade de fibras e vitaminas adequada. A presença de conservantes, corantes e outras substâncias sintéticas, associada com uma dieta composta por alimentos ricos em açúcar, pode refletir numa redução de ácidos graxos de cadeia curta, como o butirato, sintetizado pelas bactérias que compõem a microbiota intestinal e servem como substrato de forma a favorecer alterações intestinais (OLIVEIRA, 2019).

O tratamento para a constipação consiste na educação dos pacientes por meio de esclarecimentos sobre horários e posicionamento corretos para evacuação. Os horários devem preferencialmente acompanhar o reflexo gastrocólico intestinal, que consiste no aumento da motilidade colônica após a alimentação e é mais intenso pela manhã. Por isso, convém estabelecer um horário fixo para a ida ao banheiro, preferencialmente pela manhã, vinte minutos após o desjejum com posicionamento adequado, aproveitando esse reflexo matinal (AMARAL et al., 2012).

O aumento de fibras na dieta é considerado um tratamento de primeira linha para a constipação, pois as fibras ingeridas podem influenciar os perfis da microbiota fecal, causar alterações no complexo ambiente gastrointestinal e promover a proliferação de bactérias, sobretudo as benéficas. Os oligossacarídeos, incluindo frutooligossacarídeos e galactooligossacarídeos, inulina e possivelmente outras fibras solúveis, são, portanto, considerados prebióticos que podem estimular a proliferação preferencial de lactobacilos, bifidobactérias e outras bactérias saudáveis no cólon, regularizando o trânsito intestinal (WGO, 2018).

As limitações deste estudo decorrem do pequeno tamanho da amostra e pelo número reduzido de casos de constipação, impedindo verificar associações mais fortes entre os marcadores de alimentação saudável e o quadro de constipação. Além disso, a presença de professores apenas da rede pública tornou a amostra ainda menos representativa e pode constituir um viés nos resultados. 


\section{CONCLUSÃO}

A maioria dos professores estudados apresentou consumo adequado de frutas e baixo de industrializados, mas precisam aumentar a ingestão de verduras e legumes, feijões e reduzir os doces. A prevalência de professores que apresentam constipação intestinal foi baixa e, mesmo sendo observadas inadequações alimentares, só foi possível observar associação da presença de constipação com o consumo inadequado de industrializados e feijão. Outras alterações intestinais observadas, ainda que também em pequeno número, foram sintomas parciais de constipação, com risco para desenvolver o quadro ou potencialmente benéficas indicando uma melhora no quadro evacuatório. São necessários novos estudos mais detalhados a fim de compreender os fatores que impedem o consumo alimentar adequado dos professores, a fim de manter a saúde intestinal e o bom funcionamento do organismo como um todo.

\section{Nota do Autor:}

Agradeço a bolsa de iniciação científica do Centro Universitário Senac, aos professores orientadores, amigos e família.

\section{REFERÊNCIAS}

AMARAL, Ana Paula Garcez et al. Incidência de constipação intestinal em estudantes de nível superior do sexo feminino. Fisioterapia Brasil - Volume 13 - Número 5 setembro/outubro de 2012. Disponível em:

hhttps://portalatlanticaeditora.com.br/index.php/fisioterapiabrasil/article/download/569 /II70/3 225> Acesso em 4 de Outubro de 2021

ASBRAN - ASSOCIÇÃO BRASILEIRA DE NUTRIÇÃO. Percepções de professores da educação básica sobre alimentação saudável e educação alimentar e nutricional na escola. R. Assoc. bras. Nutr. 202I; I2 (2): 2I-4I ISSN 2357-7894. Disponível em: < https://www.rasbran.com.br/rasbran/article/view/2063> Acesso em I8 de Novembro de 2021

BRASIL. Ministério da Saúde. Guia alimentar para a população brasileira: promovendo a alimentação saudável. Normas e manuais técnicos. Brasília, 2014.

Disponível

em:

〈http://nutricao.saude.gov.br/docs/geral/guia_alimentar_conteudo.pdf〉 Acesso em I8 de Novembro de 2021

CARVAlHO, Ana Paula Lambrecht de. ZANARDO, Vivian Polachini Skzypek. Consumo de água e outros líquidos em adultos e idosos residentes no Município de Erechim- Rio Grande do Sul. PERSPECTIVA, Erechim. v.34, n.125, p. I17-126, março/2oıo.

Disponível em:

https://www.uricer.edu.br/site/pdfs/perspectiva/I25_79.pdf>

Acesso em 6 de Dezembro de 202I. 
CARVALHO, Maria Regina Viveiros de. Perfil do professor da educação básica / Maria Regina Viveiros de Carvalho. - Brasília, DF : Instituto Nacional de Estudos e Pesquisas Educacionais Anísio Teixeira, 2018.67 p. - (Série Documental. Relatos de Pesquisa, ISSN or40-655I ; n. 4I) Disponível em:

〈http://portal.inep.gov.br/documents/r86968/486324/Perfil+do+Professor+da+Educa\% $\mathrm{C}_{3}$ $\% \mathrm{~A}_{7} \quad \% \mathrm{C}_{3} \% \mathrm{~A}_{30}+\mathrm{B} \% \mathrm{C}_{3} \% \mathrm{~A}_{1}$ sica/6b636752-855f-4402-b7d7-b9a43ccffd 3e?version=I.13> Acesso em 4 de Outubro de 202I

CARUSO, Lúcia. Distúrbios do trato digestório. In: CUPPARI, Lilian. Nutrição clínica no adulto. $4^{\circ}$ Edição. Barueri- SP Editora Manole, 2019. p. 283.

CHEFFER, Natalha Maria. MICALISKI, Emerson Liomar. A correlação entre hábitos de vida e o nível de estresse dos docentes de uma escola pública de Palmitinho/RS. Revista Saúde e desenvolvimento.Vol. il n.6. Jan-Mar -2oi6

CRUZ, Roberto Moraes et al. Retorno ao trabalho? Indicadores de saúde mental em professores durante a pandemia da Covid-i9. Polyphonía, v. 3I/I, jan.-jun. 2020.p.327-328. Disponível em: <https://www.mendeley.com/reference-manager/reader/213b7c255dda-3afo-b273c49dc8646re7/7dr6f3b8-3592-c2od-6372-898abd4c6rige>

Acesso em io de Maio de 202I

DEMOLINER, Fernanda; DALTOÉ, Luciane. COVID-ı9: nutrição e comportamento alimentar no contexto da pandemia. Perspectiva: Ciência e Saúde, Osório, V. 5 (2): 36-50, Ago 2020. Disponível em: 〈http://sys.facos.edu.br/ojs/index.php/perspectiva/article/view/510/405〉

Acesso em 7 de Dezembro de 2020

DUNCAN et al. Doenças crônicas não transmissíveis no Brasil: prioridade para enfrentamento e investigação. Artigos. Rev. Saúde Pública 46 (suppl I). Dez 2012. Disponível em: https://www.scielo.br/j/rsp/a/WJqKxczd7dnYmzhvVdFMgyd/?lang=pt\#> Acesso em 17 de Dezembro de 2021

EFSA- European Food Safety Authority. Scientific Opinion on Dietary Reference Values for water. EFSA Journal 2010; 8(3):1459. Parma, Italy. Disponível em: 〈https://efsa.onlinelibrary.wiley.com/doi/epdf/ro.2903/j.efsa.2010.1459〉

Acesso em ro de Maio de 202I

FERREIRA, Andréa Tereza Brito. A MULHER E O MAGISTÉRIO: RAZÕES DA SUPREMACIA FEMININA (A PROFISSÃO DOCENTE EM UMA PERSPECTIVA HISTÓRICA). Tóp. Educ .. Recife. v./6. n"' I-3. p..JJ-6I. 1998 Disponível em: 
〈https://periodicos.ufpe.br/revistas/topicoseducacionais/article/download/22455/r864I > Acesso em 4 de Outubro de 202I

FREITAS, Henrique, OLIVEIRA, Mirian, SACCOL, Amarolinda, MOSCAROLA, Jean. O método de pesquisa Survey. Revista de Administração da Universidade de São

Paulo, v. 35, n. 3, 2000. Disponível em: < http://www.clam.org.br/bibliotecadigital/uploads/publicacoes/II38_I86I_freitashenriquer ausp.pdf $>$ Acesso em 5 de Outubro de 202I

GALLINA, Luciara Souza et al. Hábito alimentar do professor: importante elemento para a promoção da saúde no ambiente escolar. Rev. Simbio-Logias, V.6, n.9, Dez/2or3. Disponível em:

https://www.ibb.unesp.br/Home/ensino/departamentos/educacao/revistasimbiologias/ habito_alimentar_do_professor.pdf> Acesso em I8 de Novembro de 202I

INEP- INSTITUTO DE PESQUISA NACIONAL E EDUCAÇÃO. Perfil do professor de educação básica, 2018. Disponível em: < https://www.gov.br/mec/pt$\mathrm{br} /$ assuntos/noticias/conheca-operfil-dos-professores-brasileiros>

Acesso em 17 de Novembro de 2021.

JAQUES, Rhaylla Maria Pio Leal et al Perfil sociodemográfico e de eliminações intestinais em mulheres atendidas em um serviço de saúde. ESTIMA, Braz. J. Enterostomal Ther., I8: eo82o, 2020. https://doi.org/I0.30886/ estima.vi8.830_PT

LAUFFER, Adriana. Efeito do estresse agudo, crônico e ambos combinados na permeabilidade intestinal de ratos. Universidade Federal do Rio Grande do Sul. Programa de Pós-graduação: Ciências em Gastroenterologia e Hepatologia. Janeiro de 2015. Disponível em:

<https://lume.ufrgs.br/handle/ıor83/r43409\#: :text=Introdu\%C3\%A 7\%C3\%A30\%3A\%200 \%2oestresse\%2opsicol\% $\mathrm{C}_{3} \% \mathrm{~B}_{3}$ gico\%2oaumenta,sintomas\%2oem\%2odist\% $\mathrm{C}_{3} \% \mathrm{BArbios} \% 2$ oga strintestinais\%2ofuncionais> Acesso em I8 de Novembro de 2021

LANDIM, Keytiani Secundo Duarte et al. Consumo alimentar, estilo e qualidade de vida de professores do Ensino Técnico. Research, Society and Development, v. Io, n. 3, eI3110312878, 2021. Disponível em: < https://rsdjournal.org/index.php/rsd/article/download/I2878/I1818/172381 > Acesso em 18 de Novembro de 2021

LEAL, Joana. Inserção da mulher no mercado de trabalho foi passo importante para novas configurações sociais. Ano: 49 - Edição №: 20 - Sociedade - Instituto de Psicologia, 2016. Disponível em: $\langle$ http://www.usp.br/aunantigo/exibir? $i d=7501 \&$ ed $=1302 \& f=23>$

Acesso em 4 de Outubro de 202I 
MALTA, Deborah carvalho et al. A pandemia da COVID-ıg e as mudanças no estilo de vida dos brasileiros adultos: um estudo transversal, 2020. Epidemiol. Serv. Saúde, Brasília, 29(4):e2020407, 2020. Disponível em: 〈https://www.scielosp.org/pdf/ress/2020.v29n4/e2020407/pt〉

Acesso em 7 de Dezembro de 2020

MAYNARD, Dayanne da Costa Anjos et al. Consumo alimentar e ansiedade da população adulta durante a pandemia do COVID-ı9 no Brasil. Pesquisa, Sociedade e Desenvolvimento , [S. l.] , v. 9, n. II, pág. e4279I19905, 2020. DOI: 10.33448 / rsdv9i1r.9905. Disponível em: 〈https://rsdjournal.org/index.php/rsd/article/view/9905〉 Acesso em 6 de março de 202I.

NOLAN, Laurence J; JENKINE, Steve M. Food addiction is associated with irrational beliefs via trait anxiety and emotional eating. Nutrients, 2019 ,II, I7II, doi I0.3390/nuiro8izir. Disponivel em: <https://www.mendeley.com/referencemanager/reader/e13621eo-If22-3cff84co-3e5036269fd9/oooaf3eb-9032-4c65-oc347eer25coesba> Acesso em 6 de Março de 2021

OLIVEIRA, Ana Filipa Canastra. Relação entre dieta, microbiota intestinal, permeabilidade intestinal e doença de Crohn. Universidade de Lisboa. Mestrado integrado em medicina. Julho de 2019. Disponível em: <

https://repositorio.ul.pt/bitstream/ro45I/427II/I/AnaCOliveira.pdf>

Acesso em 18 de Novembro de 2021

OLIVEIRA, Natália Chagas de et al. Alimentação e modulação intestinal Intestinal feeding and modulation. Braz. J. of Develop., Curitiba, v. 6, n. 9, p.66488-66498, sep. 2020. Disponível em: 〈https://www.mendeley.com/reference-manager/reader/bf826596c350-3bod-8359a94f758f6ffe/6126b774-721e-a647-4ae9-733fo4794666> Acesso em 6 de Março de 2021

PASSOS, Maria do Carmo Friche; MORAES-FILHO, Joaquim Prado. Microbiota intestinal em doenças digestivas. Arq. Gastroenterol. , São Paulo, v. 54, n. 3, pág. 255-262, julho de 2017. Disponível em <http://www.scielo.br/scielo.php?script=sci_arttext\&pid=Sooo428032017000300255\&lng=en\&nrm=iso > Acesso em io de Maio de 2021

PONTI, Mayra Antonelli et al.Efeitos da pandemia de Covid-I9 no Brasil e em Portugal: estresse peritraumático. Revista Psicologia em Pesquisa. v.i4, n 4, p. 240-257, 2020. Disponível em: 〈https://periodicos.ufjf.br/index.php/psicologiaempesquisa Acesso em 7 de Dezembro de 2020

SARAIVA, Flávia Renata de Sousa et al. Depressão e disbiose.Nutrição Brasil; 2019;18(3);175-8. Doi: 10.33233/nb.vi8i3.3522 Disponível em: 
https://portalatlanticaeditora.com.br/index.php/nutricaobrasil/article/view/3522 Acesso em 28 de Junho de 2021.

SBAN- SOCIEDADE BRASILEIRA DE ALIMENTAÇÃO E NUTRIÇÃO Água, Hidratação e Saúde. Organizadores: Azevedo, Paula Schmidt et al. Material cientifico, 2016. Disponível em: < http://sban.cloudpainel.com.br/source/Agua-HidrataAAo-eSaAde_Nestle_.pdf $>$ Acesso em I8 de Novembro de 202I.

TIERMAN et al. Nutrição Comportamental no tratamento dos transtornos alimentares. In: Alvarenga et al. Nutrição comportamental $2^{\circ}$ ed. Revisada e atualizada, Barueri, Editora Manole, 2019. p. 40I-406.

WGO - World Gastroenterology Organisation Global Guidelines. Constipação: uma perspectiva mundial, 2010. Disponível em: https://www.worldgastroenterology.org/UserFiles/file/guidelines/constipationportuguese2010.pdf> Acesso em Io de maio de 202I

WGO - World Gastroenterology Organisation Global Guidelines. Dieta e intestino, 2018. Disponível em:

https://www.worldgastroenterology.org/UserFiles/file/guidelines/diet-andthe-gutportuguese.pdf $>$ Acesso em I8 de Novembro de 2021.

WHO - World Health Organization. Diet, nutrition and the prevention of chronic diseases. Report of a joint WHO/FAO expert consultation. Geneva; 2003. (WHO Technical Report Series 916). 